OPEN ACCESS

Edited by:

Jong-Min Kim,

Seoul National University Bundang

Hospital, South Korea

Reviewed by:

Carl E. Stafstrom, Johns Hopkins Medicine

United States

Anna Tagliabue,

University of Pavia, Italy

${ }^{*}$ Correspondence:

Masahito Kawamura Jr.

m.kawamura@jikei.ac.jp

${ }^{\dagger}$ These authors have contributed equally to this work

Specialty section:

This article was submitted to Neuroenergetics, Nutrition and Brain

Health,

a section of the journal

Frontiers in Neuroscience

Received: 05 December 2020

Accepted: 17 February 2021

Published: 19 March 2021

Citation:

Takeuchi F, Nishikata N, Nishimura $M$, Nagao $K$ and

Kawamura Jr M (2021)

Leucine-Enriched Essential Amino Acids Enhance the Antiseizure Effects

of the Ketogenic Diet in Rats.

Front. Neurosci. 15:637288.

doi: 10.3389/fnins.2021.637288

\section{Leucine-Enriched Essential Amino Acids Enhance the Antiseizure Effects of the Ketogenic Diet in Rats}

\author{
Fumika Takeuchi ${ }^{1 \dagger}$, Natsumi Nishikata ${ }^{1 \dagger}$, Mai Nishimura ${ }^{1}$, Kenji Nagao $^{1}$ and \\ Masahito Kawamura Jr. ${ }^{2 *}$ \\ ${ }^{1}$ Research Institute for Bioscience Products and Fine Chemicals, Ajinomoto Co., Inc., Kawasaki, Japan, ${ }^{2}$ Department \\ of Pharmacology, Jikei University School of Medicine, Minato-ku, Japan
}

The classic ketogenic diet (KD) can be used successfully to treat medically refractory epilepsy. However, the KD reduces seizures in 50-70\% of patients with medically refractory epilepsy, and its antiseizure effect is limited. In the current study, we developed a new modified KD containing leucine (Leu)-enriched essential amino acids. Compared with a normal KD, the Leu-enriched essential amino acid-supplemented KD did not change the levels of ketosis and glucose but enhanced the inhibition of bicucullineinduced seizure-like bursting in extracellular recordings of acute hippocampal slices from rats. The enhancement of antiseizure effects induced by the addition of Leu-enriched essential amino acids to the KD was almost completely suppressed by a selective antagonist of adenosine $A_{1}$ receptors or a selective dose of pannexin channel blocker. The addition of Leu-enriched essential amino acids to a normal diet did not induce any antiseizure effects. These findings indicate that the enhancement of the antiseizure effects of the $K D$ is mediated by the pannexin channel-adenosine $A_{1}$ receptor pathway. We also analyzed amino acid profiles in the plasma and hippocampus. A normal KD altered the levels of many amino acids in both the plasma and hippocampus. The addition of Leu-enriched essential amino acids to a KD further increased and decreased the levels of several amino acids, such as threonine, histidine, and serine, suggesting that altered metabolism and utilization of amino acids may play a role in its antiseizure effects. A KD supplemented with Leu-enriched essential amino acids may be a new therapeutic option for patients with epilepsy, including medically refractory epilepsy.

Keywords: epilepsy, adenosine A1 receptors, acute brain slices, hippocampus, AminoL40

\section{INTRODUCTION}

The classic ketogenic diet (KD) is a high-fat low-carbohydrate diet therapy designed in the 1920s (Wilder, 1921). The KD induces ketosis and alters metabolism in the brain. This alteration in brain metabolism induces antiseizure effects, and $\mathrm{KD}$ can be used successfully to treat patients with pediatric epilepsy. The KD has also been identified as a useful therapy for antiepileptic drugresistant epilepsy (Hallbook et al., 2007). Despite almost 100 years of clinical use, the mechanisms underlying the antiseizure effects of the KD are not fully understood (Rho and Stafstrom, 2012; Danial et al., 2013; Simeone et al., 2018). In recent decades, several lines of evidence have revealed 
the molecular mechanisms of the $\mathrm{KD}$, including ATP-sensitive potassium ( $\mathrm{K}_{\mathrm{ATP}}$ ) channels (Ma et al., 2007; Kawamura et al., 2010), BCL-2-associated agonists of cell death (Gimenez-Cassina et al., 2012), vesicular glutamate transporters (Juge et al., 2010), lactate dehydrogenase (Sada et al., 2015), and adenosine $A_{1}$ receptors ( $\left.\mathrm{A}_{1} \mathrm{Rs}\right)$ (Masino et al., 2011; Kawamura et al., 2014). Although the KD exerts antiseizure effects through various molecular pathways, the efficacy of $\mathrm{KD}$ is still limited. It has been reported that the $\mathrm{KD}$ causes a $50 \%$ reduction in seizures in $50-70 \%$ of patients with medically refractory epilepsy (Freeman et al., 2006; Caraballo et al., 2011; Wiemer-Kruel et al., 2017; Kossoff et al., 2018). Therefore, methods to further enhance the efficacy of KD therapy are required for improving the management of epilepsy.

One possible strategy for enhancing the efficacy of the KD therapy is modifying the diet with ketogenic amino acids, such as leucine (Leu). Among the $20 \mathrm{~L}$-amino acids that make up natural proteins, nine are considered nutritionally essential. These essential amino acids may play key roles in regulating brain energy and neurotransmitter metabolism when added to the KD. Branched-chain amino acids (BCAAs), such as Leu, are readily transported across the blood-brain barrier and are also known to play an important role in neurotransmitter metabolism (Hutson et al., 2001). Several studies have assessed the modulatory effects of amino acids on the antiseizure effects of the KD. The addition of BCAAs (Leu:Ile:Val = 9:6:5) to the KD has been reported to reduce seizures in patients with medically refractory epilepsy (Evangeliou et al., 2009). It has been reported that a new combined diet with a low ketogenic ratio (KR) diet composed of specific fatty acids, low-glycemic-index carbohydrates, and high BCAAs protects against seizures to the same extent as the classic KD in a chronic kainate mouse model of epilepsy (Dallerac et al., 2017). In another study, both L- and D-leucine protected mice when administered prior to the onset of seizures induced by kainic aid injection, and D-leucine potently terminated seizures even after the onset of seizure activity (Hartman et al., 2015). These diets use varying combinations of amino acids, and there is very little understanding about the role of these amino acids in the $\mathrm{KD}$. Additionally, when modulating the diet with amino acids, it is important to take overall amino acid balance into consideration because animals detect amino acid balance and alter food intake in response to protein deficiency or excess, and amino acid imbalance causes diet aversion and affects growth (Morrison et al., 2012). Thus, we hypothesized that Leu-enriched essential amino acids, comprising all nine essential amino acids, may enhance the efficacy of the antiseizure effects of KD.

\footnotetext{
Abbreviations: KD, ketogenic diet; $\mathrm{CD}$, control diet; BCAAs, branched-chain amino acids; TCA cycle, tricarboxylic acid cycle; $A_{1} R$, adenosine $A_{1}$ receptor; $\mathrm{K}_{\mathrm{ATP}}$ channels, ATP-sensitive potassium channels; DPCPX, 8-cyclopentyl-1,3dipropylxanthine; CBX, carbenoxolone; Trp, tryptophan; His, histidine; Gln, glutamine; Glu, glutamic acid; Met, methionine; Asn, asparagine; Tyr, tyrosine; Arg, arginine; Cit, citrulline; Val, valine; Leu, leucine; Phe, phenylalanine; Thr, threonine; Lys, lysine; Ile, isoleucine; Ala, alanine; Orn, ornithine; Gly, glycine; Pro, proline; Ser, serine; $\alpha \mathrm{AAA}, \alpha$-aminoadipic acid; $\alpha \mathrm{ABA}, \alpha$-aminobutyric acid; Ala, alanine; Asp, aspartic acid; Car, carnitine; Cysthi, cystathionine; GABA, gammaaminobutyric acid; HyPro, hydroxyproline; Sar, sarcosine; Tau, taurine; Cys2, cystine; 1-MeHis, 1-methylhistidine; 3-MeHis, 3-methylhistidine.
}

In the current study, we tested two different amino acid mixtures: (1) a Leu-enriched essential amino acid mixture containing $40 \%$ Leu, hereinafter referred to as "AminoL40", and (2) a casein composition amino acid mixture (CaseinAA) as a control. AminoL40 is an amino acid mixture that was formulated to optimize muscle protein synthesis. It has been demonstrated that $1.5 \mathrm{~g}$ of AminoL40 stimulates muscle protein synthesis to the same extent as $40 \mathrm{~g}$ of whey protein (Wilkinson et al., 2018). Amino acids are categorized into glucogenic amino acids, ketogenic amino acids, or both ketogenic and glucogenic amino acids according to the fate of their degradation products. AminoL40 contains approximately $57 \%$ ketogenic amino acids and $27 \%$ ketogenic and glucogenic amino acids, while CaseinAA contains only $18 \%$ ketogenic amino acids and $20 \%$ ketogenic and glucogenic amino acids (Supplementary Figure S1). Ketogenic amino acids are degraded directly into acetyl-CoA, which is the precursor of ketone bodies. The high content of ketogenic amino acids in AminoL40 may be beneficial for sustaining ketosis while providing additional nutritional components for protein synthesis. We used this mixture and compared the effects of AminoL40-supplemented KD and CaseinAA-supplemented KD by biochemical and electrophysiological methods and assessed plasma and hippocampal amino acid profiles.

\section{MATERIALS AND METHODS}

\section{Animals}

The protocol for these animal experiments was reviewed and approved by the Institutional Animal Care and Use Committee of Jikei University and the Animal Care Committee of Ajinomoto Co., Inc. and conforms to the Guidelines for the Proper Conduct of Animal Experiments of the Science Council of Japan (2006). Sprague-Dawley (SD) rats aged 4-5 weeks were obtained from Sankyo Laboratory Service Co., Ltd. (Shizuoka, Japan). All rats were housed in colony cages and maintained on a 12:12 h light/dark cycle with free access to water.

\section{Diets}

We prepared a CRF-1 diet (Oriental Yeast Co., Ltd., Tokyo, Japan) as a control diet (CD) and four types of KDs: (1) a normal $\mathrm{KD}$ without addition of any amino acid (normal KD), (2) a KD with a $3.3 \%$ CaseinAA mixture (KD + CA), (3) a KD with a $3.3 \%$ AminoL40 mixture (KD $+3.3 \% \mathrm{AL})$, and (4) a KD with a $2.5 \%$ AminoL40 mixture (KD $+2.5 \% \mathrm{AL})$. The compositions of these diets are shown in Supplementary Table S1. The KR was calculated using Woodyatt's formula (Woodyatt, 1921), which considers the ketogenic macronutrients represented by portions of protein and fat versus glucogenic macronutrients instead of calculating the simple KR using fat: (protein + carbohydrate). Woodyatt's KR of all types of KD was maintained between 4.8:1 and 5.3:1 (Supplementary Table S1). To evaluate the effect of AminoL40 alone, a non-ketogenic diet based on AIN-93G was prepared with a $3.3 \%$ AminoL40 mixture (CD + 3.3\% AL). The proportions of each amino acid in the CaseinAA and AminoL40 mixture are shown in Supplementary Table S2. 


\section{Food Intake and Body Weight Measurements and Tissue Sampling}

Food intake and body weight were measured twice a week. Sampling of the liver, hippocampus, and blood was performed immediately after treatment under isoflurane anesthesia; the animals were placed in the acrylic induction chamber filled with a mixture of air and isoflurane, and then isoflurane was administered via a mask. The liver and hippocampus were weighed and stored at $-80^{\circ} \mathrm{C}$ until analysis. Blood samples were collected in tubes on ice containing ethylenediaminetetraacetate acid (EDTA). Plasma samples were obtained by centrifuging the blood samples.

\section{Blood Biochemistry}

Blood glucose and ketone body levels in blood samples collected from a small needle stick of the tail vein were measured using FreeStyle Optium Neo blood glucose meters (Abbott Diabetes Care, Witney, Oxfordshire, United Kingdom) and FreeStyle Optium $\beta$-ketone Test Strips (Abbott Diabetes Care, Witney, Oxfordshire, United Kingdom). Lactate and insulin concentrations in venous plasma samples were measured using the Lactate Colorimetric Assay Kit II (BioVision Inc., Milpitas, CA, United States) and Morinaga Rat Insulin Measurement Kit (Morinaga Biochemical Research Laboratory, Yokohama, Japan).

\section{Quantification of Amino Acid and Metabolite Concentrations}

The plasma sample was mixed with the internal standard solution (stable isotope-labeled amino acids in water) and deproteinized with acetonitrile. Frozen hippocampal tissue was powdered using a Multi-Beads Shocker (Yasui Kikai, Osaka, Japan) and homogenized in an ice-cold methanol aqueous solution containing L-phenyl-d5-alanine. The homogenate was further mixed with water and chloroform, and the upper phase was dried. The residue was dissolved in water and mixed with the internal standard solution. The plasma and hippocampal samples were derivatized with APDSTAG ${ }^{\circledR}$ (FUJIFILM Wako Pure Chemicals, Osaka, Japan) and analyzed using liquid chromatography coupled with tandem mass spectrometry (LC-MS/MS) as described in a previous publication (Shimbo et al., 2009).

\section{Extracellular Recordings}

Male SD rats aged 4-5 weeks were fed a $\mathrm{CD}, \mathrm{KD}+\mathrm{CA}$, $\mathrm{KD}+2.5 \% \mathrm{AL}, \mathrm{KD}+3.3 \% \mathrm{AL}$, or $\mathrm{CD}+3.3 \% \mathrm{AL}$ for 13-16 days. Rats were anesthetized with isoflurane $(5 \%$ in air) and decapitated. Standard slice preparation was performed, and standard recording conditions were employed for field recordings as described in our previous works (Kawamura et al., 2014; Oyama et al., 2020). Briefly, four to five coronal hippocampal slices of $400-\mu \mathrm{m}$ thickness were made in ice-cold artificial cerebrospinal fluid (aCSF) containing (in $\mathrm{mM}$ ) 126 $\mathrm{NaCl}, 3 \mathrm{KCl}, 1.5 \mathrm{MgCl}_{2}, 2.4 \mathrm{CaCl}_{2}, 1.2 \mathrm{NaH}_{2} \mathrm{PO}_{4}, 11$ glucose, and $26 \mathrm{NaHCO}_{3}$ (osmolarity 320 mOsm, pH 7.4 when saturated with $95 \% \mathrm{O}_{2}+5 \% \mathrm{CO}_{2}$ ) using a vibrating slice cutter (PRO 7, Dosaka EM, Kyoto, Japan). The slices were incubated in aCSF saturated with $95 \% \mathrm{O}_{2}+5 \% \mathrm{CO}_{2}$ for $30-40 \mathrm{~min}$ at $37^{\circ} \mathrm{C}$, then kept at room temperature until recording. The slices were then placed on a nylon net in the recording chamber and submerged in and continuously superfused with aCSF at a flow rate of $2 \mathrm{ml} \mathrm{min}^{-1}$ at $32 \pm 0.5^{\circ} \mathrm{C}$ using a thermostatic controller (TC-324C, Warner Instruments, Hamden, CT, United States).

Field population spikes (PSs) were recorded using the same method as described in our previous works (Kawamura et al., 2014). Briefly, medium wall $(1.5 \mathrm{~mm})$ capillary filament glass was pulled on a Sutter P-97 micropipette puller (Novato, CA, United States), resulting in electrode resistances of 8-12 $\mathrm{M} \Omega$. A recording electrode filled with $3 \mathrm{M} \mathrm{NaCl}$ was placed in the pyramidal layer of the CA3 region. A twisted, bipolarinsulated tungsten electrode was placed in the hilus of the dentate gyrus as a stimulating electrode; stimuli were delivered at 30 $\mathrm{s}$ intervals, the pulse duration was $100 \mu \mathrm{s}$, and the intensity was adjusted such that the amplitude of evoked PS responses was between 0.6 and $1.4 \mathrm{mV}$. Seizure-like activity was induced by blocking GABAergic inhibition with bicuculline $(10 \mu \mathrm{M})$. KD-induced inhibition of seizure-like activity is sensitive to glucose level (Kawamura et al., 2014). In the current method, the antiseizure effects of acute hippocampal slices were observed by changing extracellular glucose concentration from brain slicemaintenance level $(11 \mathrm{mM})$ to normoglycemic level in the hippocampus (3 mM, Hu and Wilson, 1997). Antiseizure effects were measured by comparing the area under the curve $(\mathrm{mV} / \mathrm{ms}$, "area" in Figure 3A) of bicuculline-induced bursting 1 min before and $25 \mathrm{~min}$ after changing the concentration of extracellular glucose concentration. PSs were recorded via an AC amplifier (Model 3000, A-M Systems, Carlsborg, WA, United States) and filtered at $1 \mathrm{kHz}$. The data were digitized (16-channel A/D board, National Instruments Japan, Tokyo, Japan) at a rate of $4 \mathrm{kHz}$ and analyzed online using custom NeuroAcquisition software (Galtware, Denver, CO, United States). All area data of bicuculline-induced bursting were normalized to the baseline (\% of baseline) and are expressed as the mean \pm standard error (SEM), as described in our previous publication (Kawamura et al., 2014). The graphs in the figures show sparse markers every four points. For the extracellular recordings, the time point indicated as the onset of changing glucose concentration is the calculated time when the solution first began to mix into the aCSF in the slice chamber.

\section{Drug Applications}

Bicuculline, 8-cyclopentyl-1,3-dipropylxanthine (DPCPX) and carbenoxolone (CBX) were purchased from Sigma (St. Louis, MO, United States). As described in our previous publication (Oyama et al., 2020), all drugs were dissolved in aCSF at 100 times the desired final concentration and then applied via a syringe pump (STC-525, Terumo, Tokyo, Japan) to achieve the final concentration. For pretreatment, bicuculline, DPCPX, or $\mathrm{CBX}$ was superfused for at least $30 \mathrm{~min}$ before changing extracellular glucose. After application of any antagonist, no additional recordings were performed from the same slice.

\section{Statistical Analysis}

The data were compared by using unpaired $t$-tests for two groups and by one-way analysis of variance (ANOVA) with Bonferroni's 
correction for three or more groups using GraphPad InStat 3.10 (GraphPad Software, La Jolla, CA, United States) or R 3.6.0 (R Core Team, 2019, R Foundation for Statistical. Computing, Vienna, Austria). $P$-values $(P)$ less than 0.05 were considered statistically significant.

\section{RESULTS}

\section{Body Weight and Blood Biochemistry}

We fed a $\mathrm{CD}$, normal $\mathrm{KD}, \mathrm{KD}+\mathrm{CA}, \mathrm{KD}+2.5 \% \mathrm{AL}$, or $\mathrm{KD}+3.3 \% \mathrm{AL}$ to rats for $2-3$ weeks. We did not find that any of the diets had severe adverse effects during this period. We first collected basic data for amino acid-supplemented KD-fed animals. The food intake (kcal/day) of rats fed a normal $\mathrm{KD}$ was significantly lower than that of rats fed a $\mathrm{CD}$, but there was no difference among normal the $\mathrm{KD}, \mathrm{KD}+\mathrm{CA}, \mathrm{KD}+2.5 \%$ $\mathrm{AL}$, and $\mathrm{KD}+3.3 \% \mathrm{AL}$ groups (Figure 1C), indicating that the addition of CaseinAA or AminoL40 to the KD did not affect the food intake of rats. All KD groups exhibited a deceleration of weight gain compared with CD (Figures 1A,B), which is similar to previous findings (Ruskin et al., 2009). The body weight of the $\mathrm{KD}+\mathrm{CA}$ group was significantly higher than that of the normal KD group at 21 days after diet treatment (Figure 1B) but not different from that of the KD $+2.5 \% \mathrm{AL}$ or $\mathrm{KD}+3.3 \%$ $\mathrm{AL}$ groups. We also measured the liver weight for each group. The liver weights were significantly lower in the KD groups than in the CD group (Figure 1D), which is also similar to previous findings (Thio et al., 2006). The liver weights corrected for body weight (liver/body weights) of the normal $\mathrm{KD}, \mathrm{KD}+\mathrm{CA}$, or $\mathrm{KD}+2.5 \% \mathrm{AL}$ group were not different from those of the $\mathrm{CD}$ group (Figure 1E), indicating that the reduction in liver weight might have been caused by the deceleration of weight gain. The liver/body weight of the $\mathrm{KD}+3.3 \% \mathrm{AL}$ group was significantly lower than that of the $\mathrm{CD}$ and $\mathrm{KD}+\mathrm{CA}$ groups, but the difference was small (Figure 1E). Compared with CD, blood ketone levels were elevated in normal KD diets (Figure 2B) and was within the range of physiological ketosis observed under a sustained KD (Phillips, 2019). Normal KD group exhibited a significant decrease in blood glucose concentration and a decrease in blood insulin concentration compared with the $\mathrm{CD}$ group (Figures 2A,C). The levels of glucose, ketone bodies, and insulin did not differ between the $\mathrm{KD}, \mathrm{KD}+\mathrm{CA}, \mathrm{KD}+2.5 \%$ $\mathrm{AL}$, and $\mathrm{KD}+3.3 \% \mathrm{AL}$ groups (Figures 2A-C). There was no difference in blood lactate concentrations between any of the groups, including the CD group (Figure 2D). These results suggest that adding amino acids to the $\mathrm{KD}$ does not induce significant differences in food intake, body weight, liver weight, or blood biochemistry.

\section{AminoL40 Enhanced the Antiseizure Effects of Ketogenic Diet}

We investigated the antiseizure effect of AminoL40supplemented KD. We have previously reported that bicuculline-induced seizure-like bursting in acute hippocampal slices from normal $\mathrm{KD}$-fed rodents is suppressed by reducing the extracellular glucose concentration from brain slice-maintaining levels of glucose $(11 \mathrm{mM})$ to physiological levels of glucose (3 mM) (Kawamura et al., 2014). The effect of the normal KD continued to be observed in acute brain slices for up to $5 \mathrm{~h}$ after decapitation. This method is easy and useful for comparing the efficacy of antiseizure effects because it directly measures the inhibition rate of seizure-like bursting. Therefore, we used the same method to compare the antiseizure effects of $\mathrm{KD}+\mathrm{CA}$ and $\mathrm{KD}+\mathrm{AL}$. We recorded PSs in the pyramidal layer of the CA3 region in acute hippocampal slices from rats in all groups. All recorded slices exhibited seizure-like bursting upon bicuculline $(10 \mu \mathrm{M})$ application. Changing the extracellular glucose concentration from 11 to $3 \mathrm{mM}$ induced inhibition of bicuculline-induced bursting in slices from $\mathrm{KD}+\mathrm{CA}$-fed rats but not in those from CD-fed rats (Figure 3). The efficacy of the inhibitory effect of $\mathrm{KD}+\mathrm{CA}$ on bursting was not different from that of the normal KD (Supplementary Figure S2), indicating that adding CaseinAA to KD does not change the efficacy of the antiseizure effect of $\mathrm{KD}$. KD $+2.5 \% \mathrm{AL}$ did not significantly change the inhibition rate of bursting suppression compared with KD + CA (Figure 3). However, KD + 3.3\% AL significantly enhanced bursting inhibition (Figure 3). These results indicate that $3.3 \% \mathrm{AL}$, but not $3.3 \% \mathrm{CA}$, enhances the efficacy of the antiseizure effects of $\mathrm{KD}$ in acute hippocampal slices from rats.

\section{Involvement of $A_{1} R$ in the Antiseizure Effects of AminoL40-Supplemented Ketogenic Diet}

We next examined whether AminoL40 itself generates any antiseizure effects. A normal diet supplemented with AminoL40 $(\mathrm{CD}+3.3 \% \mathrm{AL})$ did not induce suppression of bicucullineinduced bursting in hippocampal slices (Figure 4A1-A2), suggesting that AminoL40 itself does not have an antiseizure effect on its own. Previously, we reported that KD-induced inhibition of bursting in hippocampal slices is caused by activation of $\mathrm{A}_{1}$ Rs following ATP release from pannexin-1 channels and its breakdown to adenosine (Kawamura et al., 2010, 2014). The inhibition of bursting of KD was suppressed by the $\mathrm{A}_{1} \mathrm{R}$-selective antagonist DPCPX and did not occur in slices from $A_{1}$ R-deficient mice. A low dose of CBX (10 $\mu \mathrm{M}$, which inhibits pannexin channels but not connexin channels) and a pannexin-1-specific peptide antagonist suppressed the inhibition of bursting in slices from KD-fed rats (Kawamura et al., 2014). In the current study, DPCPX (300 nM) significantly suppressed the inhibition of bicuculline-induced bursting in hippocampal slices from $\mathrm{KD}+3.3 \%$ AL-fed rats (Figure 4B1-B2). A low dose of CBX $(10 \mu \mathrm{M})$ also suppressed bursting inhibition in hippocampal slices from $\mathrm{KD}+3.3 \% \mathrm{AL}$-fed rats (Figure 4C1-C2). These results suggest that the enhancement of antiseizure effects by $\mathrm{KD}+3.3 \% \mathrm{AL}$ is mediated by an increase in $\mathrm{A}_{1} \mathrm{R}$ activation through an increase in ATP release from pannexin channels.

\section{Amino Acid Profiles in the Plasma and Hippocampus}

Finally, we examined the amino acid profiles of $\mathrm{CD}-$, normal $\mathrm{KD}-, \mathrm{KD}+\mathrm{CA}-, \mathrm{KD}+2.5 \% \mathrm{AL}-$ and $\mathrm{KD}+3.3 \% \mathrm{AL}-$ fed rats. We measured the concentrations of amino acids and 
A

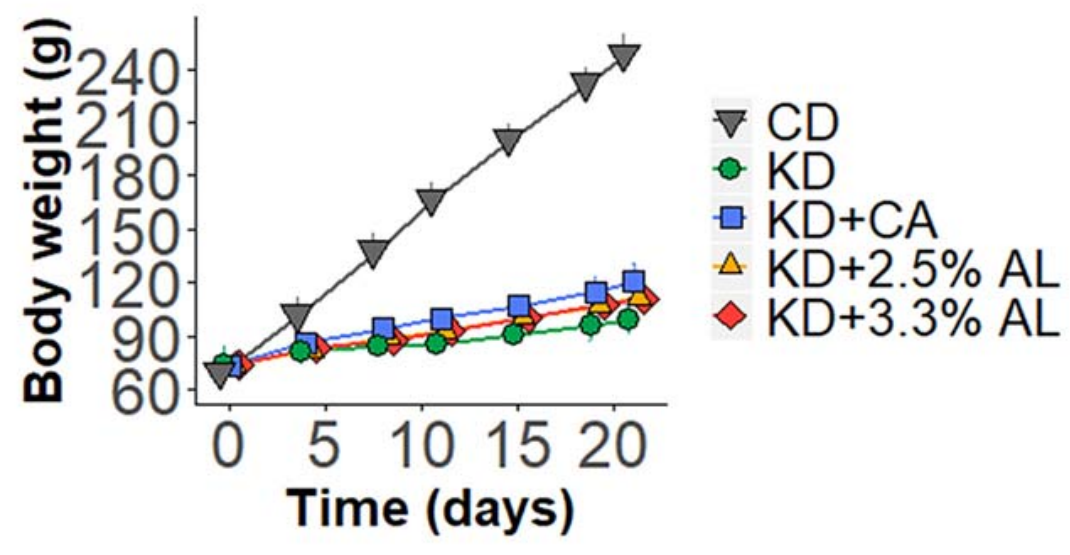

B

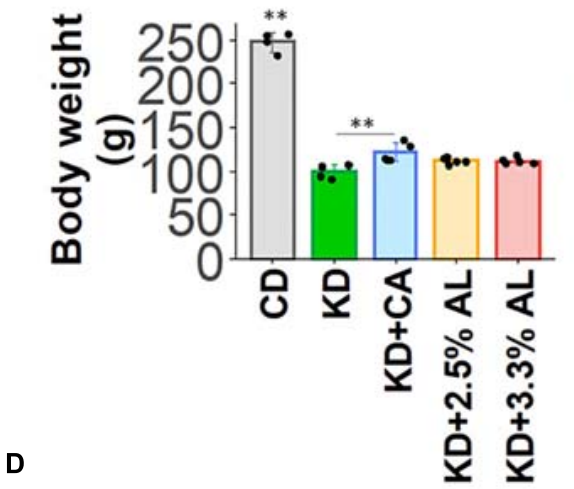

C
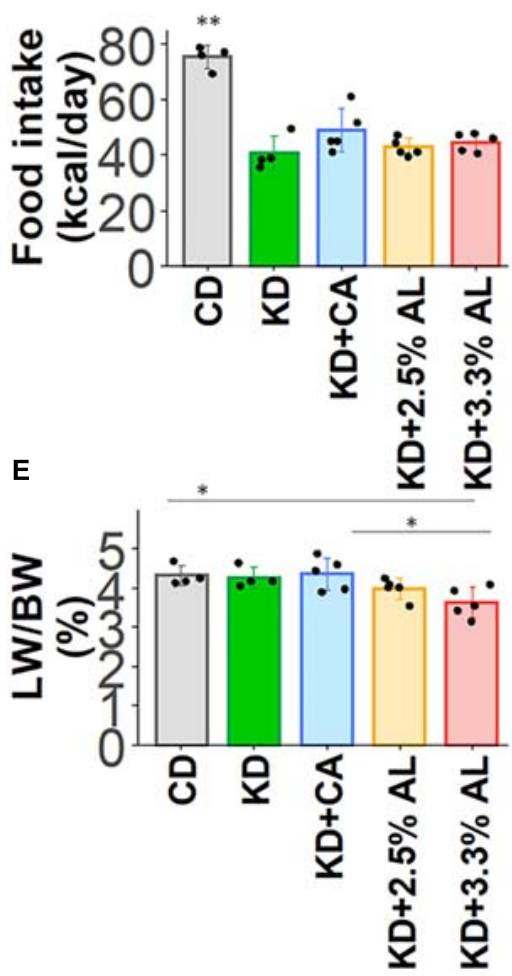

FIGURE 1 | (A) Time course of the growth of control diet (CD)-fed, normal ketogenic diet (KD)-fed, KD + CA-fed, KD + 2.5\% AL-fed, and KD + 3.3\% AL-fed rats. (B) Summary of average body weight $(\mathrm{g})$ at 21 days after feeding. $F(4,18)=259.682, P<0.0001$; one-way analysis of variance (ANOVA) with post hoc test. (C) Summary of average food intake (kcal/day) at 21 days after feeding. $F(4,18)=30.934, P<0.0001$; one-way ANOVA with post hoc test. (D) Summary of average liver weight $(\mathrm{g})$ at 21 days after feeding. $F(4,18)=120.589, P<0.0001$; one-way ANOVA with post hoc test. (E) Summary of average liver weight relative to body weight (\%). $F(4,18)=3.948, P<0.05$; one-way ANOVA with post hoc test; $\mathrm{CD}, n=4$; normal $\mathrm{KD}, n=4 ; \mathrm{KD}+\mathrm{CA}, n=5 ; \mathrm{KD}+2.5 \% \mathrm{AL}, n=5 ; \mathrm{KD}+3.3 \%$ AL, $n=5 ; * P<0.05 ; * * P<0.01$.

their metabolites in the plasma and hippocampus. Interestingly, compared with the $\mathrm{CD}$, the normal KD altered the levels of many amino acids both in the plasma and hippocampus, generating a distinct amino acid pattern. The addition of CaseinAA to KD did not change the overall amino acid pattern, while further changes were observed upon the addition of AminoL40 (Figure 5A). The levels of all amino acids and metabolites were compared, and compared with the $\mathrm{CD}$, the normal $\mathrm{KD}$ increased the mean levels of serine (Ser), asparagine (Asn), histidine (His), lysine (Lys), proline (Pro), ornithine (Orn), glutamine (Gln), and aABA in the plasma (Supplementary Table S3) and the levels of Ser, Asn, His, Lys, Gln, glycine (Gly), Car, cystathionine (Cysthi), and alpha-aminobutyric acid (aABA) in the hippocampus (Supplementary Table S4) and decreased the levels of tryptophan 

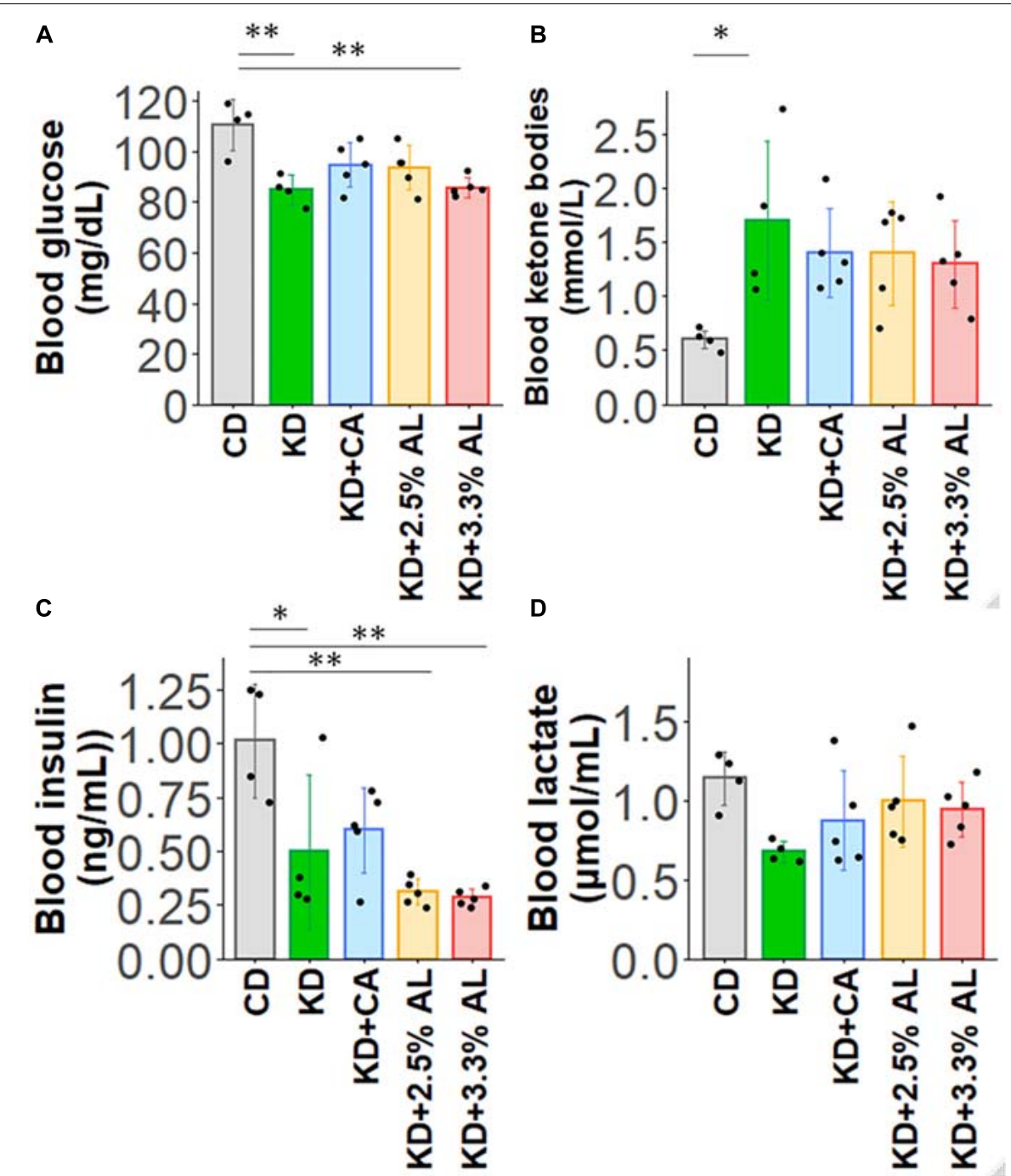

FIGURE 2 | (A) Summary of the average blood glucose concentrations ( $\mathrm{mg} / \mathrm{dll}$ ) of CD-fed, normal KD-fed, KD + CA-fed, KD + 2.5\% AL-fed, and KD + 3.3\% AL-fed rats at 21 days after feeding. $F(4,18)=7.274, P<0.01$; one-way ANOVA with post hoc test. (B) Summary of average blood ketone body levels (mmol/L) at 21 days after feeding. $F(4,18)=3.113, P<0.05$; one-way ANOVA with post hoc test. (C) Summary of average blood insulin levels $(\mathrm{ng} / \mathrm{ml}) . F(4,18)=8.7,08, P<0.001$; one-way ANOVA with post hoc test. (D) Summary of average blood lactate levels $(\mu \mathrm{mol} / \mathrm{ml}) . F(4,18)=2.229, P=0.107$; one-way ANOVA with post hoc test. CD, $n=4$; normal KD, $n=4 ; \mathrm{KD}+\mathrm{CA}, n=5 ; \mathrm{KD}+2.5 \% \mathrm{AL}, n=5 ; \mathrm{KD}+3.3 \% \mathrm{AL}, n=5 ;{ }^{*} P<0.05 ;{ }^{* *} P<0.01$.

(Trp), methionine (Met), tyrosine (Tyr), phenylalanine (Phe), Gly, alanine (Ala), cystine (Cys2), hydroxyproline, and taurine (Tau) in the plasma (Supplementary Table S3) and the levels of Met, arginine (Arg), and hydroxyproline in the hippocampus (Supplementary Table S4). Compared with the normal KD, both $\mathrm{KD}+2.5 \% \mathrm{AL}$ and $\mathrm{KD}+3.3 \% \mathrm{AL}$ increased the mean levels of threonine (Thr) in the plasma and the levels of Thr and carnitine (Car) in the hippocampus (Figure 5E) and decreased the mean levels of His, Ser, and sarcosine (Sar) in the plasma and the levels of His, Ser, Gln, valine (Val), and aABA in the hippocampus (Supplementary Table S4). At the higher dose of AminoL40 (3.3\%), additional changes were observed: increased levels of Trp, decreased levels of Ser and His in the plasma, and decreased mean levels of Phe in the hippocampus (Supplementary Table S4). Despite the additional intake of BCAAs included in AminoL40, rats fed $\mathrm{KD}+2.5 \% \mathrm{AL}$ and $\mathrm{KD}+3.3 \% \mathrm{AL}$ did not exhibit increased plasma and hippocampal levels of Leu, isoleucine (Ile), Val, and Lys compared with those fed the KD (Figures 5B-F).

\section{DISCUSSION}

In the current study, we developed a new modified KD containing leucine (Leu)-enriched essential amino acids (AminoL40). We compared the inhibitory effect of each ketogenic diet on bicuculline-induced seizure-like bursting as well as blood chemistry and amino acid profiles. Compared with normal $\mathrm{KD}$, AminoL40-supplemented $\mathrm{KD}(\mathrm{KD}+3.3 \% \mathrm{AL})$ did not change blood biochemistry. However, AminoL40-supplemented $\mathrm{KD}$ increased the inhibition rate of seizure-like bursting in 


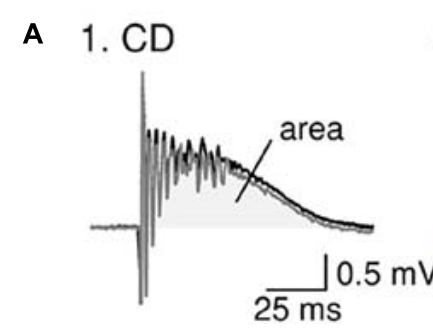

\section{2. $\mathrm{KD}+\mathrm{CA}=$ a) before}

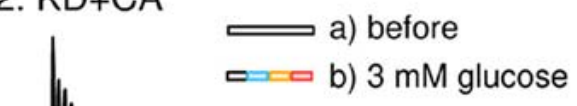

3. $\mathrm{KD}+2.5 \% \mathrm{AL}$

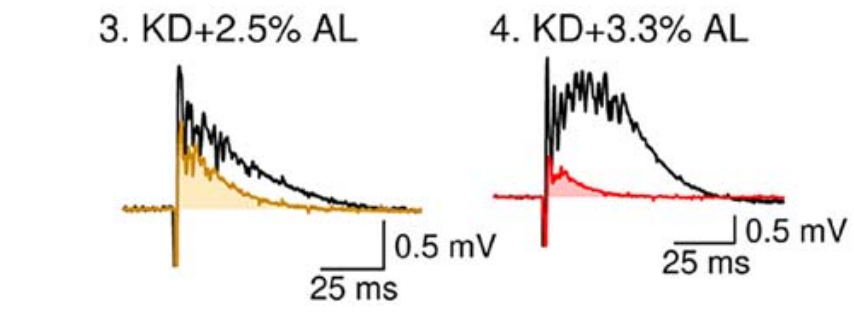

4. $\mathrm{KD}+3.3 \% \mathrm{AL}$

$25 \mathrm{~ms}$

B
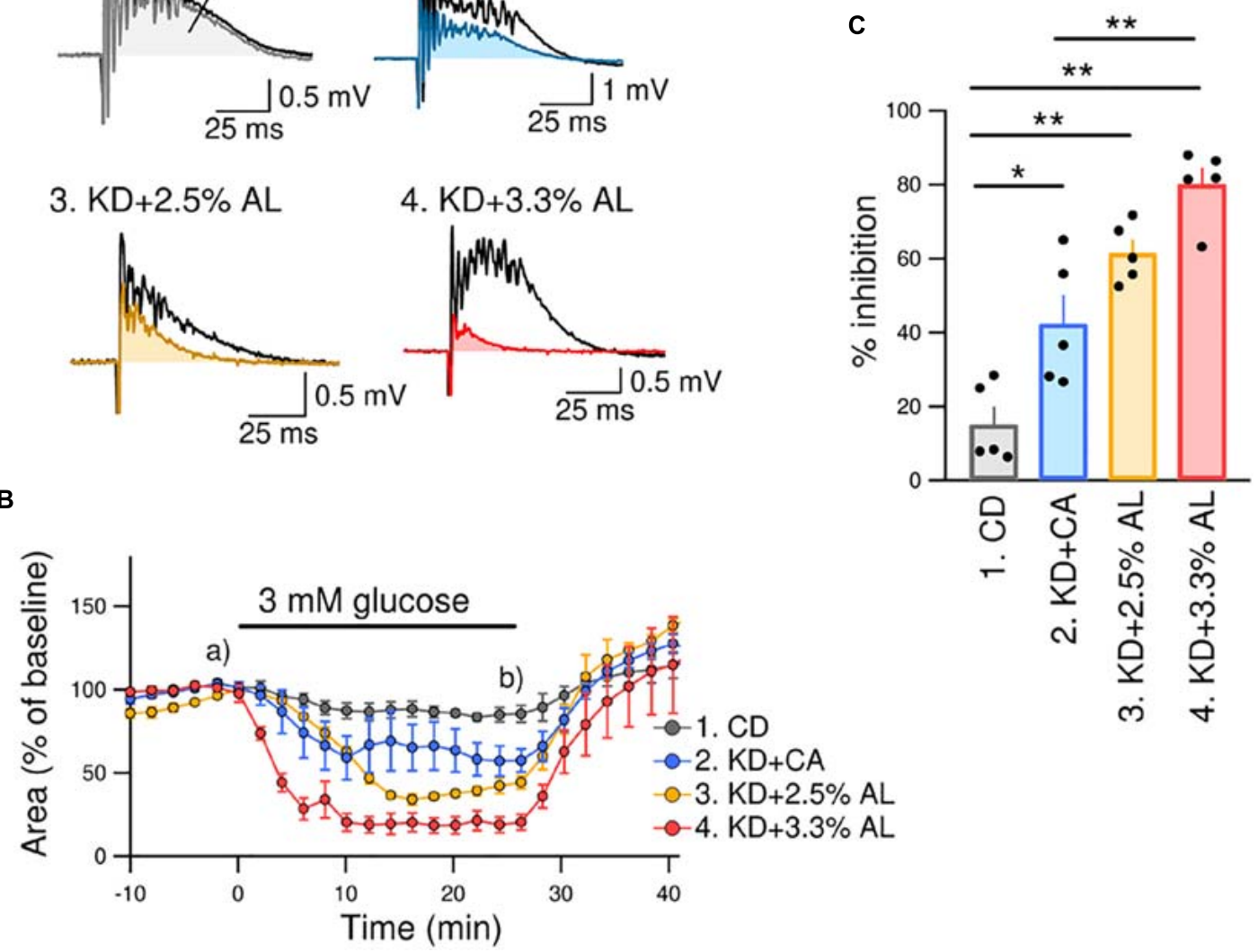

FIGURE 3 | KD + 3.3\% AL enhanced the antiseizure effect on bicuculline-induced bursting. (A) Traces of bicuculline (10 $\mu \mathrm{M})$-induced bursting in acute hippocampal slices from CD-fed (1), KD + CA-fed (2), KD + 2.5\% AL-fed (3), and KD + 3.3\% AL-fed rats (4). Black traces show seizure-like bursts 1 min before changing the extracellular glucose concentration, and the colored traces show bursting 25 min after changing the extracellular glucose concentration from 11 to $3 \mathrm{mM}(3 \mathrm{mM}$ glucose). Both traces were taken at the points indicated in (B) ( $a$ and b). (B) Time course of the effect of $3 \mathrm{mM}$ glucose for 25 min on the area of bicuculline-induced bursting (\% of baseline). Averages of the areas are shown for each timepoint every 2 min, and the SEMs are shown as vertical bars. (C) Summary of inhibition of the area of seizure-like bursting in $3 \mathrm{mM}$ glucose in slices from CD-fed, $\mathrm{KD}+\mathrm{CA}$-fed, $\mathrm{KD}+2.5 \% \mathrm{AL}$-fed, and $\mathrm{KD}+3.3 \% \mathrm{AL}$-fed rats. $F(3,16)=27.048, P<0.0001$; one-way ANOVA with post hoc test; $\mathrm{CD}, n=5 ; \mathrm{KD}+\mathrm{CA}, n=5 ; \mathrm{KD}+2.5 \% \mathrm{AL}, n=5 ; \mathrm{KD}+3.3 \% \mathrm{AL}, n=5 ;{ }^{*} P<0.05 ;{ }^{* \star} P<0.01$.

acute hippocampal slices. The inhibitory effect of the AminoL40supplemented KD on bursting was almost completely suppressed by DPCPX and by a low dose of CBX. The addition of AminoL40 to a normal diet did not inhibit bursting, suggesting that AminoL40 itself has no antiseizure effect and that AminoL40 enhances antiseizure effects of the KD through the pannexin- $\mathrm{A}_{1} \mathrm{R}$ antiseizure pathway.

The ketogenic diet is widely acknowledged as effective in treating epilepsy, and many patients with drug-resistant seizures benefit from this non-pharmacologic option. However, its efficacy is still limited. It has been reported that the $\mathrm{KD}$ causes a $50 \%$ reduction in seizures in $50-70 \%$ of patients with medically refractory epilepsy (Freeman et al., 2006; Caraballo et al., 2011; Wiemer-Kruel et al., 2017; Kossoff et al., 2018). In the current study, we also found that normal KD inhibited seizure-like bursting only by half (Figure 3), indicating that $\mathrm{KD}$ alone achieves only limited antiseizure effects. We found that adding AminoL40 to the $\mathrm{KD}$ enhanced the antiseizure effects of the KD.

Activation of adenosine receptors is one of the key mechanisms of KD-induced antiseizure effects. The KD has been reported to reduce the frequency and duration of electrographic seizures in mice with genetically induced seizures. Such reduction is suppressed by intraperitoneal injection of the $A_{1} R$ selective antagonist DPCPX, and KD has no effect on $\mathrm{A}_{1} \mathrm{R}$-deficient mice (Masino et al., 2011). It has also been reported that exogenous ketone supplementation with ketone esters or ketone salts induces anticonvulsant effects through activation of $\mathrm{A}_{1} \mathrm{R}$ (Kovacs et al., 2017). These in vivo studies show that $\mathrm{KD}$ promotes $\mathrm{A}_{1} \mathrm{R}$-induced antiseizure effects. We also reported the antiseizure effects of $\mathrm{A}_{1} \mathrm{R}$ in an in vitro study (Kawamura et al., 2014). KD is known to increase brain ATP levels in both humans (Pan et al., 1999) and rodents (DeVivo et al., 1978; Nakazawa et al., 1983; Nylen et al., 2009), and the source of adenosine may be such 

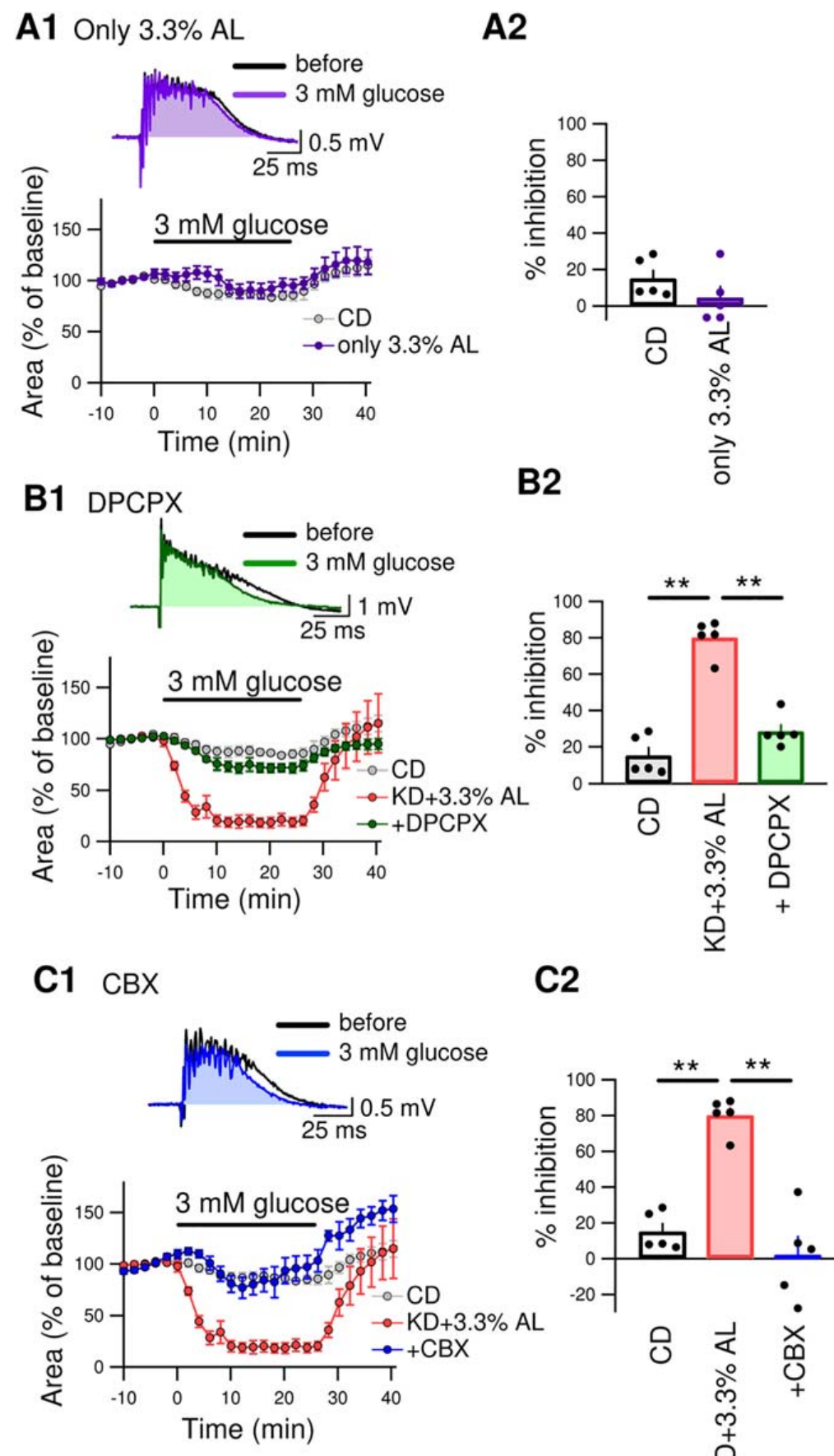

C2

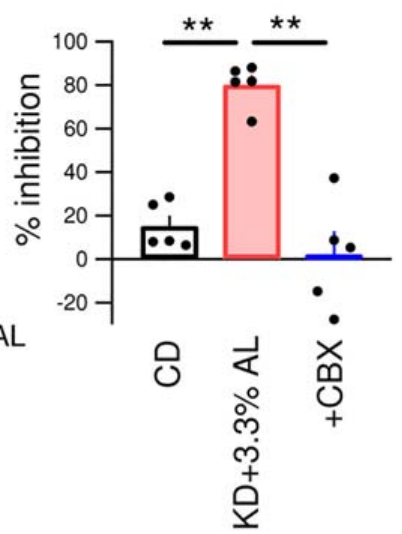

FIGURE 4 | (A1) The top traces show bicuculline-induced bursting in acute hippocampal slices from CD + 3.3\% AL-fed rats 1 min before and 25 min after changing the extracellular glucose concentration from 11 to $3 \mathrm{mM}$ ( $3 \mathrm{mM}$ glucose). The time course of the effect of $3 \mathrm{mM}$ glucose for $25 \mathrm{~min}$ on the area of bicuculline-induced bursting (\% of baseline) is shown in the lower graph. (A2) Summary of inhibition of the area of seizure-like bursting in slices from CD-and CD + 3.3\% AL-fed rats in the presence of $3 \mathrm{mM}$ glucose. $t(8)=1.312, P=0.2261$; unpaired $t$-test; $C D, n=5 ; C D+3.3 \% \mathrm{AL}, n=5$. The same data for the CD as in Figures $\mathbf{3 B}, \mathbf{C}$. (B1) The top traces show bursting in acute hippocampal slices from KD + 3.3\% AL rats in the presence of 3-dipropylxanthine (DPCPX) (300 nM) 1 min before and 25 min after changing the glucose concentration to $3 \mathrm{mM}$. The time course of the effect of $3 \mathrm{mM}$ glucose for $25 \mathrm{~min}$ on the area of bicuculline-induced bursting (\% of baseline) is shown in the lower graph. (B2) Summary of inhibition of the area of bursting in the presence of $3 \mathrm{mM}$ glucose. $F(2,12)=61.353, P<0.0001$; one-way ANOVA with post hoc test; $\mathrm{CD}, n=5 ; \mathrm{KD}+3.3 \% \mathrm{AL}, n=5 ;+\mathrm{DPCPX}, n=5 ;{ }^{\star \star} P<0.01$. The same data for the $\mathrm{CD}$ and $\mathrm{KD}+3.3 \%$ AL as in Figures $\mathbf{3 B}, \mathbf{C}$. (C1) The top traces show bursting in acute hippocampal slices from KD +3.3\% AL rats in the presence of low-dose carbenoxolone (CBX) (10 $\mu \mathrm{M}) 1$ min before and $25 \mathrm{~min}$ after changing the glucose concentration to $3 \mathrm{mM}$ glucose. The time course of the effect of $3 \mathrm{mM}$ glucose is shown in the lower graph. (C2) Summary of inhibition of the area of bursting in the presence of $3 \mathrm{mM}$ glucose. $F(2,12)=31.948, P<0.0001$; one-way ANOVA with post hoc test; $\mathrm{CD}, n=5 ; \mathrm{KD}+3.3 \% \mathrm{AL}, n=5 ;+\mathrm{CBX}$, $n=5 ;{ }^{* \star} P<0.01$. The $\mathrm{CD}$ and $\mathrm{KD}+3.3 \% \mathrm{AL}$ data are the same as those in Figures 3B,C. 
A

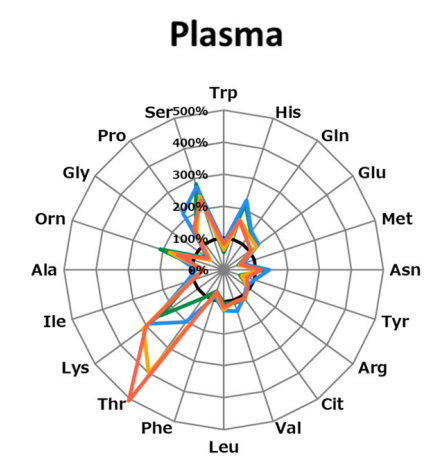

Hippocampus

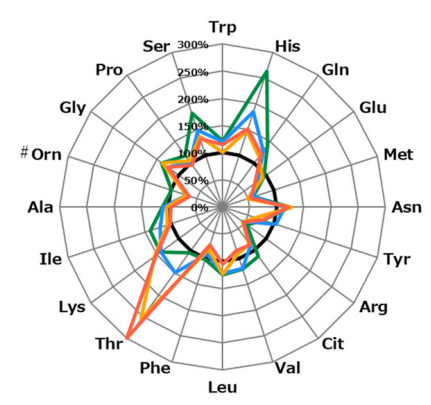

B

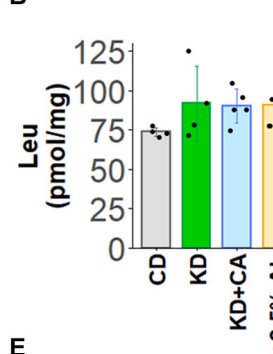

C

D

E

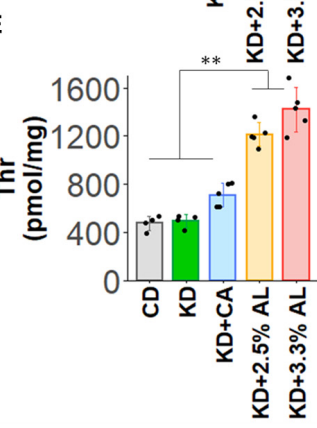

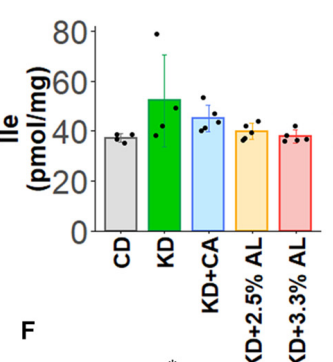

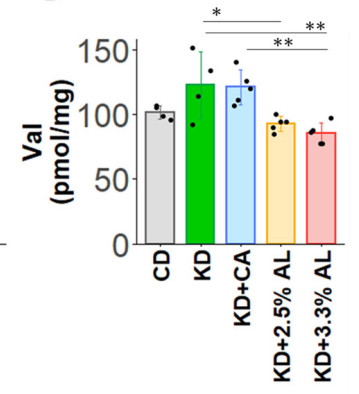

FIGURE 5 | (A) Radar charts of amino acid profiles of CD-fed, normal KD-fed, KD + CA-fed, KD + 2.5\% AL-fed and KD + 3.3\% AL-fed rats at 21 days after feeding. Results are shown for plasma (left) and hippocampus (right). The average levels of amino acids in each diet are compared with the levels in CD-fed and expressed in percentage when CD-fed rats (black) is 100\%. \#, removing outliers; Trp, tryptophan; His, histidine; Gln, glutamine; Glu, glutamic acid; Met, methionine; Asn, asparagine; Tyr, tyrosine; Arg, arginine; Cit, citrulline; Val, valine; Leu, leucine; BCAAs, branched-chain amino acids; Phe, phenylalanine; Thr, threonine; Lys, lysine; lle, isoleucine; Ala, alanine; Orn, ornithine; Gly, glycine; Pro, proline; Ser, serine. (B) Summary of the average concentration of Leu in the hippocampus (pmol/mg). F(4, $18)=1.614, P=0.214$; one-way ANOVA with post hoc test. (C) Summary of the average concentration of lle in the hippocampus (pmol/mg). $F(4,18)=2.456$, $P=0.0829$; one-way ANOVA with post hoc test. (D) Summary of the average concentration of Val in the hippocampus (pmol/mg). $F(4,18)=7.494, P<0.001$; one-way ANOVA with post hoc test. (E) Summary of the average concentration of Thr in the hippocampus (pmol/mg). $F(4,18)=66.991, P<0.0001 ;$ one-way ANOVA with post hoc test. (F) Summary of the average concentration of Lys in the hippocampus $(\mathrm{pmol} / \mathrm{mg}) . F(4,18)=3.362, P<0.05$; one-way ANOVA with post hoc test. $\mathrm{CD}, n=4$; normal $\mathrm{KD}, n=4 ; \mathrm{KD}+\mathrm{CA}, n=5 ; \mathrm{KD}+2.5 \% \mathrm{AL}, n=5 ; \mathrm{KD}+3.3 \% \mathrm{AL}, n=5 ;{ }^{*} P<0.05 ;{ }^{* *} P<0.01$.

increase in ATP levels. Our previous report showed that when the intracellular ATP concentration is increased in hippocampal CA3 pyramidal neurons, physiological levels of extracellular glucose open pannexin-1 channels and release intracellular ATP into the extracellular space. Released ATP is rapidly broken down to adenosine, and adenosine activates $A_{1} R$ leading to subsequent opening of $K_{\text {ATP }}$ channels. Opening of $K_{\text {ATP }}$ channels promotes hyperpolarization and reduces the excitability of CA3 pyramidal neurons (Kawamura et al., 2010). These reports indicate that one of the mechanisms of $\mathrm{KD}$-induced antiseizure effects is the pannexin- 1 channel- $A_{1} R-K_{A T P}$ channel pathway and involves an increase in brain ATP levels. In the current study, we found that $\mathrm{KD}+3.3 \% \mathrm{AL}$-induced inhibition of bursting was almost completely suppressed by a selective $A_{1} R$ antagonist or the selective dose of pannexin inhibitor CBX. In the absence of the $\mathrm{KD}$, AminoL40 did not induce any antiseizure effects. These findings suggest that improved efficacy of KD by AminoL40 is induced by increased activation of $\mathrm{A}_{1} \mathrm{R}$ through ATP release from pannexin-1 channels.

Adenosine receptors are well established to have therapeutic potential for neurological disorders, including epilepsy, stroke, neuropathic pain, and mental disorders (Masino et al., 2009). Recent evidence shows that the KD improves the behaviors of animal models of neuropathic pain (Ruskin et al., 2009), autism spectrum disorder (Ruskin et al., 2013), and schizophrenia (Kraeuter et al., 2015). Enrichment with AminoL40 might 
enhance the therapeutic effects of the $\mathrm{KD}$ for neurological disorders such as autism spectrum disorder, which is often comorbid with epilepsy.

We found that the addition of AminoL40 enhanced the antiseizure effects of the KD through activation of $A_{1} R$. However, the detailed mechanism regarding how the essential amino acids in AminoL40 trigger the pannexin-1 channel- $A_{1} R$ pathway when added to the $\mathrm{KD}$ is still undetermined. Our new amino acid mixture, AminoL40, contains 40\% Leu along with other essential amino acids, which are known to play key roles in brain energy and neurotransmitter metabolism. Amino acids can be metabolized to provide tricarboxylic acid (TCA) cycle intermediates and to replenish the cycle through anaplerosis (Owen et al., 2002; Sonnewald, 2014; Sperringer et al., 2017). Leu is oxidized to acetyl-CoA through acetoacetate and utilized in the TCA cycle, which is the only known pathway for the terminal oxidation of Leu. Other essential amino acids are similarly metabolized into compounds such as acetyl-CoA, succinyl-CoA, and fumarate, which can enter the TCA cycle and provide carbon skeletons (Supplementary Figure S3; Berg et al., 2002). During starvation, Gln is converted to glutamic acid (Glu) and then to $\alpha$-ketoglutarate and enters the TCA cycle, which leads to energy production (Owen et al., 2002). Additionally, Leu can activate glutamate dehydrogenase and enhance the conversion of Glu to $\alpha$-ketoglutarate, which is the rate-determining step of the TCA cycle (Wang et al., 2018; Biswas et al., 2019). Therefore, under KD feeding, which mimics starvation, amino acids may have a large effect on fueling the TCA cycle.

We assessed amino acid profiles and found that compared with a normal diet, the normal KD significantly altered both plasma and hippocampal amino acid levels. All KD groups showed elevated mean levels of Ser, Asn, His, Lys, Pro, and Gln in the plasma and elevated mean levels of Ser, Asn, His, Lys, Gln, and Gly in the hippocampus. Each of these amino acids can provide carbon skeletons for different TCA cycle intermediates. The addition of AminoL40 to the KD induced further changes in the plasma and hippocampal levels of some amino acids. Surprisingly, there were no significant increases in BCAA levels in the plasma and hippocampus, even following the addition of AminoL40. It is possible that amino acids such as BCAAs are used as energy sources in the brain as well as peripheral muscles under low-glucose conditions and that changes in amino acid profiles could not be detected. We found that Thr levels strongly correlated with the Thr content ratio in each diet, and the $\mathrm{KD}+3.3 \%$ AL group showed significantly higher Thr levels than that of other groups. Threonine dehydratase catabolizes Thr to 2-ketobutyrate, which is converted to succinyl-CoA. When levels are increased, Thr might enter the TCA cycle through the threonine dehydratase pathway and might alter ATP synthesis. The addition of a specific combination of amino acids may have supported the production of energy by replenishing TCA cycle intermediates via anaplerotic reactions, thus leading to an increase in ATP synthesis and enhancing ATP release through pannexin channels, which may have induced antiseizure effects through $\mathrm{A}_{1} \mathrm{R}$. Further studies, such as flux analysis studies, are needed to clarify the detailed mechanism of action of AminoL40 in improving brain energy metabolism.
In the current study, we found that AminoL40 enhanced the antiseizure effects of the KD. AminoL40 is composed of nine essential amino acids. Administration of AminoL40supplemented diets for the experimental period resulted in no severe adverse effects and did not change the basic peripheral effects of the normal KD, suggesting that AminoL40 can be used safely in rodents. Additional studies are needed in the future to clarify the effect of Leu-enriched essential amino acids in ketogenic diets used in clinical settings. Leuenriched essential amino acids may provide new options for the KD treatment of epilepsy, including medically refractory epilepsy.

\section{DATA AVAILABILITY STATEMENT}

The original contributions presented in the study are included in the article/Supplementary Material, further inquiries can be directed to the corresponding author/s.

\section{ETHICS STATEMENT}

The animal study was reviewed and approved by the Institutional Animal Care and Use Committee of Jikei University and the Animal Care Committee of Ajinomoto Co., Inc.

\section{AUTHOR CONTRIBUTIONS}

$\mathrm{NN}, \mathrm{KN}$, and MK conceived, designed the research, edited, and revised the manuscript. FT, MN, and MK performed the experiments and analyzed the data. FT and MK prepared the figures. FT, NN, KN, and $\mathrm{MK}$ drafted the manuscript. FT, NN, MN, KN, and MK approved the final version of manuscript. All authors contributed to the article and approved the submitted version.

\section{FUNDING}

This work was supported by the JSPS KAKENHI (grant no. 20K07745 to MK).

\section{ACKNOWLEDGMENTS}

We would like to acknowledge Dr. Susan A. Masino for the comments on this manuscript, Ms. Sachise Karakawa (Ajinomoto Co., Inc.) for the amino acid analysis, and Ms. Noriko Kawasaki (Ajinomoto Co., Inc.) for supporting the experiments.

\section{SUPPLEMENTARY MATERIAL}

The Supplementary Material for this article can be found online at: https://www.frontiersin.org/articles/10.3389/fnins.2021. 637288/full\#supplementary-material 


\section{REFERENCES}

Berg, J. M., Tymoczko, J. L., and Stryer, L. (2002). Biochemistry. New York, Ny: W.H. Freeman.

Biswas, D., Duffley, L., and Pulinilkunnil, T. (2019). Role of branched-chain amino acid-catabolizing enzymes in intertissue signaling, metabolic remodeling, and energy homeostasis. FASEB J. 33, 8711-8731. doi: 10.1096/fj.201802 842RR

Caraballo, R., Vaccarezza, M., Cersosimo, R., Rios, V., Soraru, A., Arroyo, H., et al. (2011). Long-term follow-up of the ketogenic diet for refractory epilepsy: multicenter argentinean experience in 216 pediatric patients. Seizure 20, 640645. doi: 10.1016/j.seizure.2011.06.009

Dallerac, G., Moulard, J., Benoist, J. F., Rouach, S., Auvin, S., Guilbot, A., et al. (2017). Non-ketogenic combination of nutritional strategies provides robust protection against seizures. Sci. Rep. 7:5496. doi: 10.1038/s41598-01705542-3

Danial, N. N., Hartman, A. L., Stafstrom, C. E., and Thio, L. L. (2013). How does the ketogenic diet work? Four potential mechanisms. J. Child Neurol. 28, 1027-1033. doi: 10.1177/0883073813487598

DeVivo, D. C., Leckie, M. P., Ferrendelli, J. S., and McDougal, D. B. Jr. (1978). Chronic ketosis and cerebral metabolism. Ann. Neurol. 3, 331-337. doi: 10. 1002/ana.410030410

Evangeliou, A., Spilioti, M., Doulioglou, V., Kalaidopoulou, P., Ilias, A., Skarpalezou, A., et al. (2009). Branched chain amino acids as adjunctive therapy to ketogenic diet in epilepsy: pilot study and hypothesis. J. Child Neurol. 24, 1268-1272. doi: 10.1177/0883073809336295

Freeman, J., Veggiotti, P., Lanzi, G., Tagliabue, A., and Perucca, E. (2006). The ketogenic diet: from molecular mechanisms to clinical effects. Epilepsy Res. 68, 145-180. doi: 10.1016/j.eplepsyres.2005.10.003

Gimenez-Cassina, A., Martinez-Francois, J. R., Fisher, J. K., Szlyk, B., Polak, K., Wiwczar, J., et al. (2012). BAD-dependent regulation of fuel metabolism and K(ATP) channel activity confers resistance to epileptic seizures. Neuron 74 , 719-730. doi: 10.1016/j.neuron.2012.03.032

Hallbook, T., Kohler, S., Rosen, I., and Lundgren, J. (2007). Effects of ketogenic diet on epileptiform activity in children with therapy resistant epilepsy. Epilepsy Res. 77, 134-140. doi: 10.1016/j.eplepsyres.2007.09.008

Hartman, A. L., Santos, P., O’Riordan, K. J., Stafstrom, C. E., and Hardwick, J. M. (2015). Potent anti-seizure effects of D-leucine. Neurobiol. Dis. 82, 46-53. doi: 10.1016/j.nbd.2015.05.013

Hu, Y., and Wilson, G. S. (1997). Rapid changes in local extracellular rat brain glucose observed with an in vivo glucose sensor. J. Neurochem. 68, 17451752.

Hutson, S. M., Lieth, E., and LaNoue, K. F. (2001). Function of leucine in excitatory neurotransmitter metabolism in the central nervous system. J. Nutr. 131, 846S-850S. doi: 10.1093/jn/131.3.846S

Juge, N., Gray, J. A., Omote, H., Miyaji, T., Inoue, T., Hara, C., et al. (2010). Metabolic control of vesicular glutamate transport and release. Neuron 68, 99-112. doi: 10.1016/j.neuron.2010.09.002

Kawamura, M. Jr., Ruskin, D. N., Geiger, J. D., Boison, D., and Masino, S. A. (2014). Ketogenic diet sensitizes glucose control of hippocampal excitability. J. Lipid Res. 55, 2254-2260. doi: 10.1194/jlr.M046755

Kawamura, M. Jr., Ruskin, D. N., and Masino, S. A. (2010). Metabolic autocrine regulation of neurons involves cooperation among pannexin hemichannels, adenosine receptors, and KATP channels. J. Neurosci. 30, 3886-3895. doi: 10. 1523/JNEUROSCI.0055-10.2010

Kossoff, E. H., Zupec-Kania, B. A., Auvin, S., Ballaban-Gil, K. R., Christina Bergqvist, A. G., Blackford, R., et al. (2018). Optimal clinical management of children receiving dietary therapies for epilepsy: updated recommendations of the International Ketogenic Diet Study Group. Epilepsia Open 3, 175-192. doi: 10.1002/epi4.12225

Kovacs, Z., D'Agostino, D. P., Dobolyi, A., and Ari, C. (2017). Adenosine A1 receptor antagonism abolished the anti-seizure effects of exogenous ketone supplementation in wistar albino Glaxo Rijswijk rats. Front. Mol. Neurosci. 10:235. doi: 10.3389/fnmol.2017.00235

Kraeuter, A. K., Loxton, H., Lima, B. C., Rudd, D., and Sarnyai, Z. (2015). Ketogenic diet reverses behavioral abnormalities in an acute NMDA receptor hypofunction model of schizophrenia. Schizophr. Res. 169, 491-493. doi: 10. 1016/j.schres.2015.10.041
Ma, W., Berg, J., and Yellen, G. (2007). Ketogenic diet metabolites reduce firing in central neurons by opening K(ATP) channels. J. Neurosci. 27, 3618-3625. doi: 10.1523/JNEUROSCI.0132-07.2007

Masino, S. A., Kawamura, M., Wasser, C. A., Pomeroy, L. T., and Ruskin, D. N. (2009). Adenosine, ketogenic diet and epilepsy: the emerging therapeutic relationship between metabolism and brain activity. Curr. Neuropharmacol. 7, 257-268. doi: 10.2174/157015909789152164

Masino, S. A., Li, T., Theofilas, P., Sandau, U. S., Ruskin, D. N., Fredholm, B. B., et al. (2011). A ketogenic diet suppresses seizures in mice through adenosine A(1) receptors. J. Clin. Invest. 121, 2679-2683. doi: 10.1172/JCI57813

Morrison, C. D., Reed, S. D., and Henagan, T. M. (2012). Homeostatic regulation of protein intake: in search of a mechanism. Am. J. Physiol. Regul. Integr. Comp. Physiol. 302, R917-R928. doi: 10.1152/ajpregu.00609.2011

Nakazawa, M., Kodama, S., and Matsuo, T. (1983). Effects of ketogenic diet on electroconvulsive threshold and brain contents of adenosine nucleotides. Brain Dev. 5, 375-380.

Nylen, K., Velazquez, J. L., Sayed, V., Gibson, K. M., and Burnham, W. M. (2009). The effects of a ketogenic diet on ATP concentrations and the number of hippocampal mitochondria in Aldh5a1(-/-) mice. Biochim. Biophys. Acta 1790, 208-212. doi: 10.1016/j.bbagen.2008.12.005

Owen, O. E., Kalhan, S. C., and Hanson, R. W. (2002). The key role of anaplerosis and cataplerosis for citric acid cycle function. J. Biol. Chem. 277, 30409-30412. doi: 10.1074/jbc.R200006200

Oyama, Y., Ono, K., and Kawamura, M. Jr. (2020). Mild hypothermia protects synaptic transmission from experimental ischemia through reduction in the function of nucleoside transporters in the mouse hippocampus. Neuropharmacology 163:107853. doi: 10.1016/j.neuropharm.2019.107853

Pan, J. W., Bebin, E. M., Chu, W. J., and Hetherington, H. P. (1999). Ketosis and epilepsy: 31P spectroscopic imaging at 4.1 T. Epilepsia $40,703-707$.

Phillips, M. C. L. (2019). "Ketogenic diet therapies in children and adults with epilepsy," in Epilepsy - Advances in Diagnosis and Therapy, ed. I. J. Al-Zwaini (London: IntechOpen).

R Core Team (2019). R: A Language and Environment for Statistical Computing. Vienna: R Foundation for Statistical Computing. Available online at: https: //www.R-project.org/

Rho, J. M., and Stafstrom, C. E. (2012). The ketogenic diet: what has science taught us? Epilepsy Res. 100, 210-217. doi: 10.1016/j.eplepsyres.2011.05.021

Ruskin, D. N., Kawamura, M., and Masino, S. A. (2009). Reduced pain and inflammation in juvenile and adult rats fed a ketogenic diet. PLoS One 4:e8349. doi: 10.1371/journal.pone.0008349

Ruskin, D. N., Svedova, J., Cote, J. L., Sandau, U., Rho, J. M., Kawamura, M., et al. (2013). Ketogenic diet improves core symptoms of autism in BTBR mice. PLoS One 8:e65021. doi: 10.1371/journal.pone.0065021

Sada, N., Lee, S., Katsu, T., Otsuki, T., and Inoue, T. (2015). ). Epilepsy treatment. Targeting LDH enzymes with a stiripentol analog to treat epilepsy. Science 347, 1362-1367. doi: 10.1126/science.aaa1299

Shimbo, K., Oonuki, T., Yahashi, A., Hirayama, K., and Miyano, H. (2009). Precolumn derivatization reagents for high-speed analysis of amines and amino acids in biological fluid using liquid chromatography/electrospray ionization tandem mass spectrometry. Rapid. Commun. Mass Spectrom. 23, 1483-1492. doi: $10.1002 / \mathrm{rcm} .4026$

Simeone, T. A., Simeone, K. A., Stafstrom, C. E., and Rho, J. M. (2018). Do ketone bodies mediate the anti-seizure effects of the ketogenic diet? Neuropharmacology 133, 233-241. doi: 10.1016/j.neuropharm.2018. 01.011

Sonnewald, U. (2014). Glutamate synthesis has to be matched by its degradation - where do all the carbons go? J. Neurochem. 131, 399-406. doi: 10.1111/jnc. 12812

Sperringer, J. E., Addington, A., and Hutson, S. M. (2017). Branched-chain amino acids and brain metabolism. Neurochem. Res. 42, 1697-1709. doi: 10.1007/ s11064-017-2261-5

Thio, L. L., Erbayat-Altay, E., Rensing, N., and Yamada, K. A. (2006). Leptin contributes to slower weight gain in juvenile rodents on a ketogenic diet. Pediatr. Res. 60, 413-417. doi: 10.1203/01.pdr.0000238244.54610.27

Wang, T., Yao, W., He, Q., Shao, Y., Zheng, R., and Huang, F. (2018). Lleucine stimulates glutamate dehydrogenase activity and glutamate synthesis by regulating mTORC1/SIRT4 pathway in pig liver. Anim. Nutr. 4, 329-337. doi: $10.1016 /$ j.aninu.2017.12.002 
Wiemer-Kruel, A., Haberlandt, E., Hartmann, H., Wohlrab, G., and Bast, T. (2017). Modified Atkins diet is an effective treatment for children with Doose syndrome. Epilepsia 58, 657-662. doi: 10.1111/epi. 13701

Wilder, R. M. (1921). The effects of ketonemia on the course of epilepsy. Mayo Clin. Proc. 2, 307-308.

Wilkinson, D. J., Bukhari, S. S. I., Phillips, B. E., Limb, M. C., Cegielski, J., Brook, M. S., et al. (2018). Effects of leucine-enriched essential amino acid and whey protein bolus dosing upon skeletal muscle protein synthesis at rest and after exercise in older women. Clin. Nutr. 37(6 Pt A), 2011-2021. doi: 10.1016/j.clnu. 2017.09.008

Woodyatt, R. T. (1921). Objects and method of diet adjustment in diabetes. Arch Intern. Med. 28:125. doi: 10.1001/archinte.1921.00100140002001
Conflict of Interest: FT, NN, MN, and KN were employees of Ajinomoto Co., Inc.

The remaining author declares that the research was conducted in the absence of any commercial or financial relationships that could be construed as a potential conflict of interest.

Copyright (c) 2021 Takeuchi, Nishikata, Nishimura, Nagao and Kawamura. This is an open-access article distributed under the terms of the Creative Commons Attribution License (CC BY). The use, distribution or reproduction in other forums is permitted, provided the original author(s) and the copyright owner(s) are credited and that the original publication in this journal is cited, in accordance with accepted academic practice. No use, distribution or reproduction is permitted which does not comply with these terms. 